\title{
Relation of PLGF in the first trimester to fetal SGA or IUGR
}

\author{
Ismaili Bashkim \\ IPH Special Hospital for Obstetry-Gyneacology "Mother Teresa" Chair-Skopje, Republic of North \\ Macedonia
}

\begin{abstract}
PIGF determination today is of particular clinical importance in determining fetal-placental development. Placental growth factor PIGF is a human, genetically encoded protein.

Objectives. Evaluation of the impact of PIGF in the first trimester, with the fetal SGA / IUGR.

Material and method. The study is a prospective study conducted at the Special Gynecology Hospital "Mother Teresa", Skopje, and the Clinical-Biochemical Laboratory, Institute of Immunology at the University Clinical Center Skopje R, North Macedonia, from February 2019 - September 2010. 698 pregnant women were included in the study.

Outcomes. Out of all controlled patients in the period February 2019-June 2010, a total of 698 pregnant women were studied. increase in PIGF values, in 1 or $25(25 \%)$ cases with SGA we have increased values of PIGF. PIGF level $>40 \mathrm{pg} / \mathrm{ml}$ cut-off resulted in 337 or $(48.28 \%)$ cases, and in 361 or $(51.71) \%$ cases have an increase in PIGF < cut-off $40 \mathrm{pg} / \mathrm{ml}$. Newborns with SGA/ IUGR resulted in 4 or $(0.57 \%)$ newborns, according to gestation evaluation criteria.

Results. In our study PIGF resulted in: sensitivity $75 \%$, specificity $50 \%$, PPV $75 \%$, NPV $50 \%$, P $=0.25$ and $\mathrm{R}=0.5$.

Discussions. Assessment of placental development during pregnancy is one of the most important predictors of fetal development and pre-eclampsia. In modern acupuncture protocols, new diagnostic methods are of particular importance.

Conclusions. PIGF methods have their own difficulties due to the high cost of PIGF reagents, Opportunities for further study are opened, because a new database has been formed in this area of obstetrics from clinically validated and diagnosed patients. Ohet A computerized database of these problems is formed.
\end{abstract}

Keywords: placental growth factor, SGA/IUGR

\section{INTRODUCTION}

Diagnosis of pregnancy and fetal condition is achieved through several contemporary clinical, biochemical, physical and electronic methods for establishing a fetoplacental fetal diagnosis.

These processes result in typical physiological changes that can be observed in the mother, placenta and fetus. Trophoblastic implantation and fertilization of the placenta play a crucial role in its development as an organ for the transport of food and oxygen to the fetus $(1,2)$.

As placental dysfunction occurs in the first trimester of adherence, the last decade provides the opportunity to placental markers early detection of patients at risk for acupuncture clinical disease and their association with prediction of fetal growth (3). Biomarkers include pregnancy-associated plasma
protein-A (PAPP-A), placental growth factor (PlGF), tyrosine kinase-1 (sFlt-1), soluble endoglin (sEng), activin-A and inhibin Specific protein substances of placental origin have been recently identified in pregnancy, the determination of which is of great value in the pursuit of normal and pathological pregnancy, one of which is undoubtedly PIGF. New protocols have been introduced for diagnostic and predictive clinical application (4). It is a Doppler ultrasound method of the uterine arteries, which is very predictive of the occurrence of eclampsia in the pregnancy which then has a direct impact on fetal growth. Roughly 1 in 10 babies are SGA / IUGR, the incidence of SGA / IUGR is higher in people of color than in white people. In developing countries in $1 / 3$ of the cases the native causes are $\mathrm{JH}$-gestation, while in $25 \%$ of fetal causes of 
increased in utero stagnation is hunger (5). LBW has an incidence of $8-10 \%$ in developed countries and $63 \%$ in developing countries. Pre-eclampsia complicates $4-8 \%$ of pregnancies (6). The average weight of the newborn is 2,500 to $4,000 \mathrm{~g}$ with a length of $51 \mathrm{~cm}$. The term low birth weight (LBW) refers to weight $<2,500$ grams regardless of the age of the pregnancy. Most babies weighing $<2,500 \mathrm{~g}$ give birth prematurely (are born before week 37 of pregnancy). In contrast, the terms intrauterine growth restriction (IUGR) and small for fetal gestational age (SGA) are calculated with reference to the age of pregnancy (10). SGAs are fetuses who have birth weight less than $10 \%$ for their gestational age (8). IUGR is a term used for fetuses who fail to make their intrauterine growth optimal. These children are pathologically young, consequently those children have an increased risk for neurological problems, congenital malformations, hypoglycemia, hypocalcaemia, and respiratory distress syndromes $(7,9)$. Synonymous for fetal malnutrition, chronic fetal distress, newborn with body mass in relation to SGA gestational age, increased intrauterine stagnation, (intrauterine growth retardation-IUGR (11) (see figure 1).

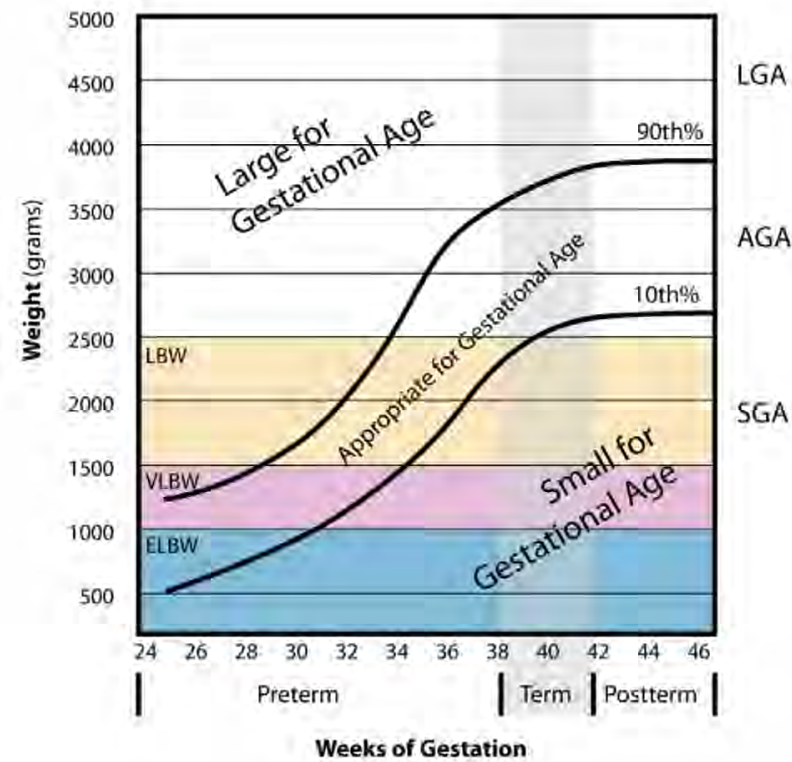

FIGURE 1. Clasification of newborn weight and weeks of gestation

Although some babies are constitutively small due to genetics (their parents are small), most SGA babies are small due to the growth problems that occur during pregnancy (12). If the baby's birth weight is below the 10th percentile for pregnancy, the baby is also SGA. It is important to note that not all SGA newborns are IUGR, they are simply younger than normal because their parents are younger $(11,13)$.

Low birth weight (LBW), a newborn with low birth weight, is defined as a newborn weighing less than 2,500 grams irrespective of gestational age (14).

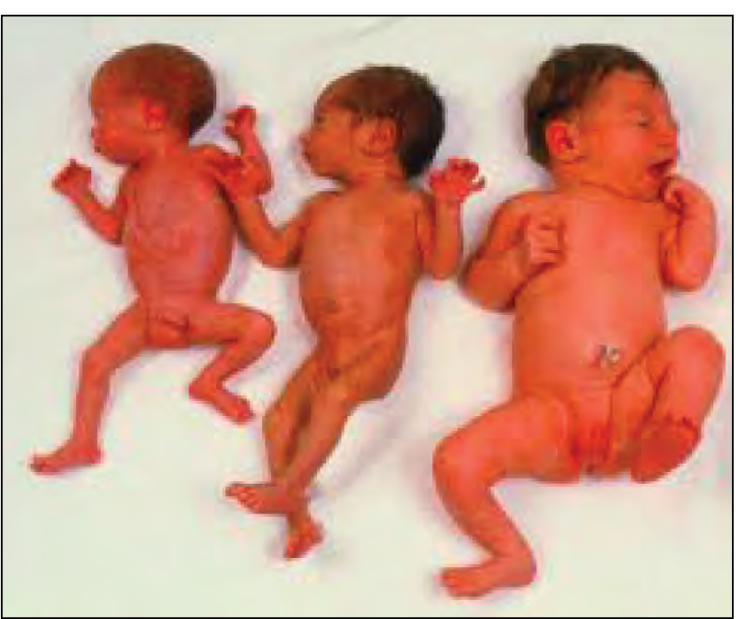

FIGURE 2. Presentation of newborn SGA in relation to normal eutrophic newborn

Accurate screening protocols exist throughout the world in the first trimester, but objective factors sometimes influence non-adherence to these protocols, such as non-screening of pregnancies, non-management of pregnancies by primary care physicians, and so on (15). As mentioned above, pre-eclampsia and increased fetal stagnation are linked (16). EPH-gestosis occurs in approximately $10-15 \%$ of first pregnancies and $5-10 \%$ of later pregnancies. Cases are diagnosed after the 34th week of pregnancy. Placental growth factor (PlGF) determination today receives particular clinical importance in determining feto-placental development. PlGF is a human protein, genetically encoded $(17,18)$

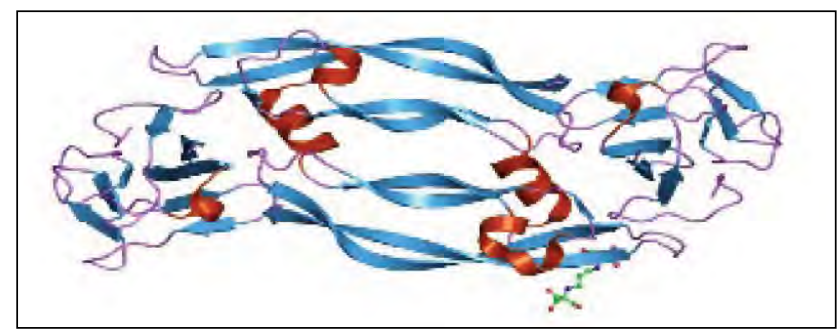

FIGURE 3. Structure of PIGF

PlGF is an angiogenic growth factor related to vascular endothelial growth factor produced exclusively by the trophoblast. In 2019, FIGO adopted PlGF as a screening method for pre-eclampsia.

In normal pregnancy, PlGF increases throughout pregnancy, reaching a peak of 26-30 weeks. 
Placental growth factor depletion in the first trimester has been consistently found during pregnancy with pre-eclampsia - eclampsia in the first trimester.

\section{OBJECTIVES}

Impact assessment of PlGF parameters in the first trimester, with SGA / IUGR fetus.

\section{MATERIALS AND METHOD}

The study is a prospective study conducted at the Special Mother-Gynecology Hospital "Mother Teresa", Skopje, and the Clinical-Biochemical Laboratory, Institute of Immunology at the University Clinical Center, Skopje R, North Macedonia, in February 2019 The study included 698 pregnant, aged 17-41 years, mean age 28.73 years \pm 3.5 months, between $11+0$ and $15+1$ week of gestation. PIGF values were calculated in the range of $3-200 \mathrm{pg} / \mathrm{ml}$, cutt-of was calculated $>40 \mathrm{pg} / \mathrm{ml}$, quantitative method of calculating PlGF values was used.

\section{STATISTICAL CALCULATION OF VALUE}

The collected data were recorded in Microsoft Access database, calculated using separate forms of Microsoft Exel 2007 and Windows 7. Computer data processing was performed with Statistica for Windows 8.0 and SPSS 8.0 for MS Windows. Test Fischer was used for group comparison $(19,20)$, the Mann-Whitney test was used to compare variables (21).

\section{RESULTS}

Out of all controlled patients in the period February 2018-February 2019, a total of 698 pregnancies were studied, mean age 28.73 years \pm 3.5 months, minimum age of study was 17 years, maximum 42 years. resulted in SGA / IUGR in 3 or $(75 \%)$ cases no increase in PlGF values, in 1 or (25\%) cases with SGA increased values of PlGF. PlGF level > cut-off $40 \mathrm{pg} / \mathrm{ml}$ resulted in 337 (48.28\%) cases, and in 361 cases (51.71\%) we have an increase in PlGF < cut-off $40 \mathrm{pg} / \mathrm{ml}$.

TABLE 1. All pregnancies in study

\begin{tabular}{|l|c|c|}
\hline Born SGAI/UGR & Total 698 & $100 \%$ \\
\hline SGA/IUGR & 4 & $0.57 \%$ \\
\hline EUTROPHIC & 694 & $99.43 \%$ \\
\hline
\end{tabular}

Graphical presentation of childrens with SGA

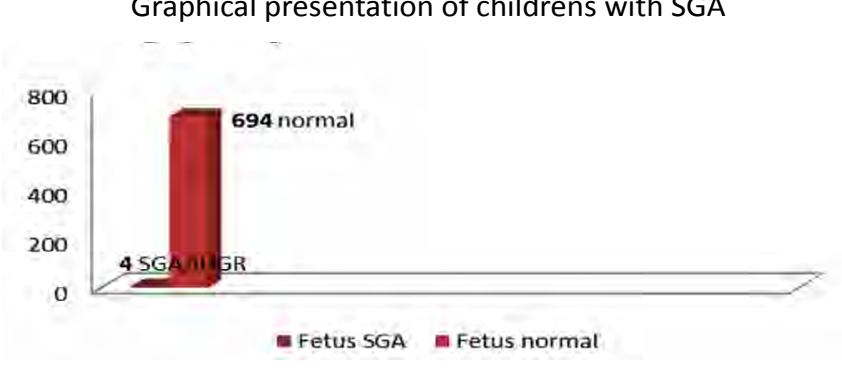

FIGURE 4. Graphical representation of all studied born

TABLE 2. Fetuses with SGA

\begin{tabular}{|l|c|c|}
\hline Fetus resulted with SGA & In total 4 SGA & $100 \%$ \\
\hline No increased values of PIGF & 3 & $75 \%$ \\
\hline Increased PIGF & 1 & $25 \%$ \\
\hline
\end{tabular}

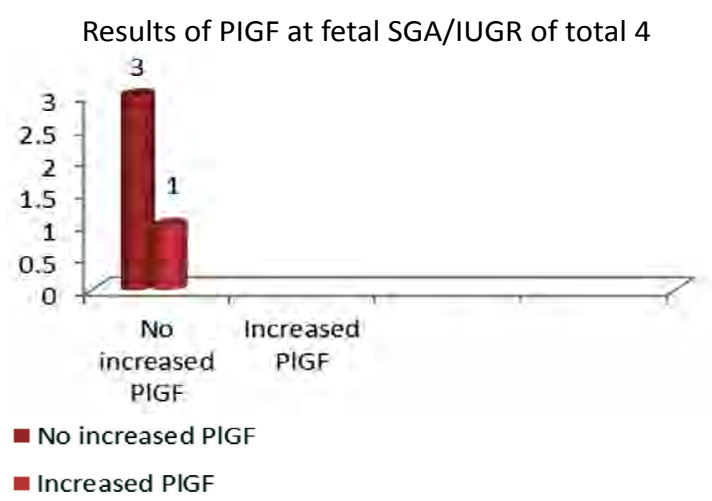

FIGURE 5. Graphical presentation of 4 cases

In our study PlGF resulted in: $75 \%$ sensitivity, $50 \%$ specificity, $75 \% \mathrm{PPV}, 50 \% \mathrm{NPV}$. $\mathrm{P}=0.25 \mathrm{R}=$ 0.5 . Confirming high reliability of PlGF early detection of pre-eclampsia and its impact on the newborn with SGA / IUGR.

\section{DISCUSSION}

Assessment of placental development during pregnancy is one of the most important predictors of fetal development and pre-eclampsia. In modern acupuncture protocols, new diagnostic methods are of particular importance. Utilizing these methods is often impossible, since their cost is high, a maximum coordination of primary and secondary level obstetricians is also required to increase diagnostic efficiency. Based on the results it is seen that in both diagnostic methods respectively PlGF it is confirmed the high predictive values of PlGF, but nonetheless that these parameters should be combined with other pregnancy diagnostic factors, and further research is needed on these two predictors of pregnancy. Fetus SGA / IUGR in 4 or $0.57 \%$. Based on the group of children to whom PIGF analysis presents results of PlGF $=>$ cut-off measure- 
ments of $40 \mathrm{pg} / \mathrm{ml}$ in 337 or (48.28\%) cases, and in 361 or $(51.71 \%)$ cases we have increased PlGF $=<$ cut-off $40 \mathrm{pg} / \mathrm{ml}$ correlation of these values with presentation of SGA / IUGR shows minimal percentage of these children. Early diagnostic data may reduce inadequate sub-hospital controls, proper and adequate monitoring of these pregnancies, reduce the length of stay or exclude the need for hospitalization.

\section{REFERENCES}

1. Hernandez, Andrade E, Brodszki J, Lingman G, Gudmundsson S, Molin J, Marsál K. Uterine artery score and perinatal outcome. Ultrasound Obstet Gynecol 2002; 19:438- 442.

2. Vergani P, Roncaglia N, Andreotti C, Arreghini A, Teruzzi M, Pezzullo JC, Ghidini A. Prognostic value of uterine artery Doppler velocimetry in growth-restricted fetuses delivered near term. Am J Obstet Gynecol 2002; 187: 932-936.

3. Kingdom J, Huppertz B, Seaward G, Kaufmann P. Development of the placental villous tree and its consequences for fetal growth. Eur $\mathrm{J}$ Obstet Gynecol Reprod Biol 2000; 92: 35-43.

4. Albaiges G, Missfelder-Lobos H, Lees C, Parra M, Nicolaides KH. One-stage screening for pregnancy complications by color Doppler assessment of the uterine arteries at 23 weeks' gestation. Obstet Gynecol 2000; 96: 559-564.

5. Lawn JE, Cousens S, Zupan J. 4 million neonatal deaths: when? Where? Why? The Lancet 2005. 365 (9462): 891-900.

6. Steegers EA, von Dadelszen P, Duvekot JJ, Pijnenborg R. Preeclampsia. Lancet. 2010; 376(9741):631-44.

7. Chamberlain G, Phillip E, Howlett BC, Masters K 1978 British births 1970. Vol 2. Obstetric care. London: William Heinemann Medical.

8. Tidwell SC, Ho HN, Chiu WH, Torry RJ, Low maternal serum levels of placenta growth factor as an antecedent of clinical preeclampsia. AmJObstet Gynecol 2001;184:1267-1272.

9. Vvan den Elzen HJ, Cohen-Overbeek TE, Grobbee DE, Quartero RW, Wladimiroff JW. Early uterine artery Doppler velocimetry and the outcome of pregnancy in women aged 35 years and older. Ultrasound Obstet Gynecol 1995; 5: 328-333.

10. Kone E, Çeka Xh, Dedja E, TW Sadler. Embriologjia mjekësore e Langman, 2013.

11. Linda J. Vorvick. Reviewed by David Zieve. Small for gestational age (SGA) at MedlinePlus. Update Date: 8/4/2009.

\section{CONCLUSIONS}

PlGF methods have their own difficulties due to the high cost of PlGF reagents, Opportunities for further study are opened, because a new database has been formed in this area of obstetrics from clinically validated and diagnosed patients. A computerized database of these problems is formed.

12. Muthayya S. Maternal nutrition \& low birth weight - what is really important? Indian Journal of Medical Research 2009, vol. 130, no. 5, pp. 600-608.

13. Vikram S Dogra, MD, Intrauterine Growth Retardation. Retrieved 2007.11-28.

14. Michael G Ross, MD, MPH, Fetal Growth Restriction. Retrieved 2010-02-25.

15. Mello $\mathrm{G}$ et al. Risk factors for fetal macrosomia: the importance of a positive oral glucose challenge test. European Journal of Endocrinology. 1997; 137:27-33.

16. Kurdi W, Campbell S, Aquilina J, England P, Harrington K. The role of color Doppler imaging of the uterine arteries at 20 weeks' gestation in stratifying antenatal care. Ultrasound Obstet Gynecol. 1998; 12: 339-345.Wiley Online Library

17. PGF placental growth factor ( Homo sapiens (human) ), GENE database, NCBI, National Center for Biotechnology Information, (https://www.ncbi.nlm.nih.gov/gene/?term=5228).

18. Maglione D, Guerriero V, Viglietto G, Ferraro MG, Aprelikova O, Alitalo K, Del Vecchio S, Lei KJ, Chou JY, Persico MG. Two alternative mRNAs coding for the angiogenic factor, placenta growth factor (PIGF), are transcribed from a single gene of chromosome 14. Oncogene 1993. 8 (4): 925-31.

19. Fisher RA. On the interpretation of $X^{2}$ from contingency tables, and the calculation of P. Journal of the Royal Statistical Society (1922) 85 (1) 8794 ..

20. Fisher, R.A. Statistical Methods for Research Workers. Oliver and Boyd. (1954). ISBN 0-05-002170-2.

21. Mann Henry B, Whitney Donald R. On a Test of Whether one of Two Random Variables is Stochastically Larger than the Other. Annals of Mathematical Statistics 1947 18(1):50-60. 